\title{
LEITO DE JORRO CÔNICO COMPOSTO DE MISTURA DE PARTÍCULAS DE AREIA E COMPÓSITO AL/PEBD
}

\author{
V. R. COVRE 1 , J. L. Z. MELO ${ }^{2}$ e M. S. BACELOS ${ }^{2}$ \\ ${ }^{1}$ Universidade Federal do Espírito Santo, Centro Universitário Norte do Espírito Santo, \\ Departamento de Engenharias e Tecnologia \\ ${ }^{2}$ Universidade Federal do Espírito Santo, Centro Universitário Norte do Espírito Santo, \\ Programa de Pós-Graduação em Energia \\ E-mail para contato: marcelo.bacelos@ufes.br
}

\begin{abstract}
RESUMO - As embalagens cartonadas Tetra Pak são compostas por camadas de papel, polietileno e alumínio. Sua reciclagem tem sido amplamente estudada, com ênfase na produção de papel e peças plásticas com o compósito AL/PEDD. Entretanto, a pirólise do compósito pode ser empregada como alternativa promissora de recuperação do alumínio e obtenção da parafina (pode ser reutilizada para produção de PEBD - matéria-prima da embalagem). Segundo a Literatura, o leito de jorro é um sistema de contato gás-sólido promissor para tal aplicação. Desta forma, esta pesquisa tem como objetivo estudar o comportamento da fluidodinâmica das partículas do compósito de polietileno e alumínio em leito de jorro cônico, utilizando areia como material inerte. As misturas de areia e compósito Al/PEBD empregadas variam em um faixa percentual de 0 a $70 \%$ de compósito. A análise dos dados de queda de pressão no leito em função da velocidade do ar permite identificar regimes de jorro estáveis para misturas com até 50\% de compósito. Os resultados mostram que a velocidade e queda de pressão de mínimo jorro diminuem com o aumento da concentração de compósito AL/PEBD no leito.
\end{abstract}

\section{INTRODUÇÃO}

O aperfeiçoamento no tratamento de resíduos sólidos tem sido tema de destaque nos últimos anos, sobretudo, pela atual tendência de produção sustentável no ramo empresarial. Neste contexto, técnicas avançadas, com finalidade de reaproveitar tais resíduos, têm sido desenvolvidas. No caso das embalagens cartonadas Tetra Pak, compostas por camadas de papel (70 a $75 \%$ ), polietileno (20 a 25 $\%$ ) e alumínio (5\%), a reciclagem do papel já é realizada eficientemente. As fibras podem ser separadas através da agitação com água em equipamentos denominados hidrapulper. A mistura remanescente de polietileno e alumínio (AL/PEBD) pode ser reaproveitada através de extrusão, preparando pellets de compósito AL/PEBD para injeção de peças moldadas (Abreu, 2002). Segundo Zuben e Neves (1999), este material possui maior resistência à tração que o polietileno de baixa densidade e, em alguns casos, pode substitui-lo.

Por outro lado, a mistura remanescente AL/PEBD possui alto valor agregado devido ao preço elevado de sua matéria prima. Neste contexto, a pirólise de compósitos AL/PEBD tem se mostrado uma 


\section{9 a 22 de outubro de 2014 \\ Florianópolis/SC}

alternativa viável na regeneração do alumínio, preservando as reservas de bauxita, e produzindo gases com potencial. A decomposição térmica do polietileno (acima de $700{ }^{\circ} \mathrm{C}$ ) em atmosfera inerte (sem oxigênio) produz uma mistura de hidrocarbonetos que pode ser utilizada para diversos fins, incluindo o uso como combustível (Buekens e Huang, 1998).

Diferentes reatores foram analisados para a aplicação da pirólise. Korkmaz et al. (2009) selecionaram um pirolisador semi-batelado de leito fixo, enquanto Haydary et al. (2013) escolheram um reator rotativo para o estudo. Marques et al. (2012) realizou um estudo sobre o potencial do leito de jorro como reator de pirólise de partículas cartonadas (discos cartonados compostos por camadas de papel, polietileno e alumínio). Marques (2013) concluiu que o leito de jorro apresenta bom contato gás sólido, e baixa segregação de partículas, sendo, portanto, uma alternativa promissora para o processamento de discos cartonados.

A fluidodinâmica do leito de jorro tem sido amplamente estudado. Choi e Meisen (1992), Olazar et al. (1992) e Bi et al. (1997) desenvolveram equações empíricas que relacionam o regime de jorro (Número de Reynolds e velocidade de mínimo jorro) para leito de jorro cônico relacionando as dimensões do equipamento com a propriedades físicas das partículas. $\mathrm{O}$ efeito de diferentes condições de operação também é uma área amplamente abordada na literatura. Wu et al. (1987) estudaram a influência da temperatura sobre a performance de leitos de jorro cônicos, e Olazar et al. (2009) complementaram o tema, adicionando a condição de baixas pressões aos seus experimentos. Bacelos (2006) avaliou o efeito do tamanho e da uniformidade do diâmetro de partícula sobre a estabilidade do regime de jorro. Melo et al. (2014) avaliaram o comportamento fluidodinâmico do compósito de polietileno e alumínio em leito de jorro a partir de experimento prático e simulação em fluidodinâmica computacional (CFD).

Desta forma, a presente pesquisa tem, como objetivo, contribuir com o estudo do leito de jorro como alternativa viável para o projeto de pirólise de resíduos AL/PEBD. Busca-se, então, avaliar o comportamento fluidodinâmico do compósito AL/PEBD ao ser misturado com partículas de sólido inerte (areia) em diferentes proporções. A adição de um material inerte de massa específica superior, porém de semelhante diâmetro de partícula garante, ao leito, maior estabilidade no regime de mínimo jorro.

\section{METODOLOGIA}

\subsection{Caracterização dos Materiais}

O material sólido do leito de jorro é composto por uma mistura de areia e compósito de alumínio e polietileno (AL/PEBD). O compósito AL/PEBD é proveniente do processo de extrusão/injeção de resíduos de polietileno e alumínio é fornecido pela empresa Tetra Pak. Na figura 1, é possível ver um exemplo de mistura com $20 \%$ em massa de compósito AL/PEBD. Nos experimentos foram utilizados misturas de 0, 10, 20, 30, 40, 50, 60 e $70 \%$ em massa de compósito AL/PEBD com areia. 


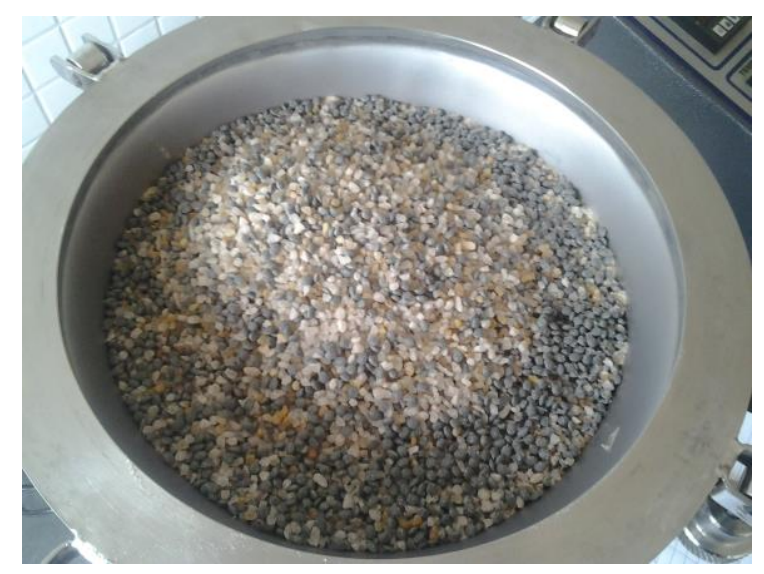

Figura 1 - Mistura com 20\% em massa de compósito AL/PEBD após utilização no leito de jorro

A massa específica do material particulado foi determinada através de picnometria. Inicialmente, o picnômetro é calibrado com água destilada. Em seguida, o mesmo é preenchido com a amostra de AL/PEBD e a massa é determinada em balança analítica (precisão de $\pm 0,5 \times 10^{-4} \mathrm{~g}$ ). Com o volume do recipiente e a massa de partículas é possível obter a massa específica aparente do leito de AL/PEBD. Na sequência, o picnômetro é, então, preenchido com querosene e sua massa específica determinada pelo mesmo procedimento. Este fluido foi escolhido pois possui massa específica conhecida e inferior à massa específica do compósito $\mathrm{AL} / \mathrm{PEBD}$, além de não apresentar interação química ou física significativa com a amostra, de forma a evitar interferência na determinação da massa específica da mistura. Novamente, a massa do sistema (AL/PEBD + querosene) é determinada e, por meio de balanço de massa no picnômetro para o compósito de AL/PEDB, é possível obter a massa específica verdadeira das amostras. Conhecendo-se o volume do picnômentro, a massa específica do querosene, das partículas de AL/PEBD, a porosidade é obtida, com base em um balanço de massa para o querosene no picnômetro.

O diâmetro das partículas selecionadas está na faixa de 2,36 a 2,80 mm. Esta seleção foi realizada através de técnica de peneiramento com peneiras de base vibratória. Desta forma, apenas partículas passantes na peneira de $2,80 \mathrm{~mm}$ e retida na peneira de $2,36 \mathrm{~mm}$ foram utilizadas neste experimento. $\mathrm{O}$ diâmetro médio das partículas é estimado através de média aritmética entre o limite superior e o limite inferior.

A Tabela 1 mostra os resultados das propriedades físicas do composto de AL/PEBD obtidas com a metodologia supracitada.

Tabela 1 - Propriedades físicas relevantes da caracterização da fase sólida

\begin{tabular}{cc}
\hline Massa específica da areia & $2340 \mathrm{~kg} / \mathrm{m}^{3}$ \\
Massa específica do compósito AL/PEBD & $828 \mathrm{~kg} / \mathrm{m}^{3}$ \\
Diâmetro médio das partículas & $0,00258 \mathrm{~m}$ \\
Porosidade da areia & 0,45 \\
Porosidade do compósito AL/PEBD & 0,48 \\
\hline
\end{tabular}




\subsection{Unidade Experimental}

Os experimentos realizados utilizaram a unidade experimental em escala piloto do PPGEN/UFES. A unidade experimental é constituída por um leito de jorro de base cônica, com ângulo de cone de $45^{\circ}$; um soprador tipo centrífugo de média pressão e vazão, da marca IBRAM, com capacidade de fornecer até $4,5 \mathrm{~m} 3 / \mathrm{min}$ de ar; um sistema de aquecimento de ar com controle PID de temperatura e um transdutor de pressão da marca Dwyer, modelo 616C-4, operando na faixa de 0 a 20 polegadas de coluna de água. $\mathrm{O}$ aparato experimental está representado esquematicamente na figura 2.
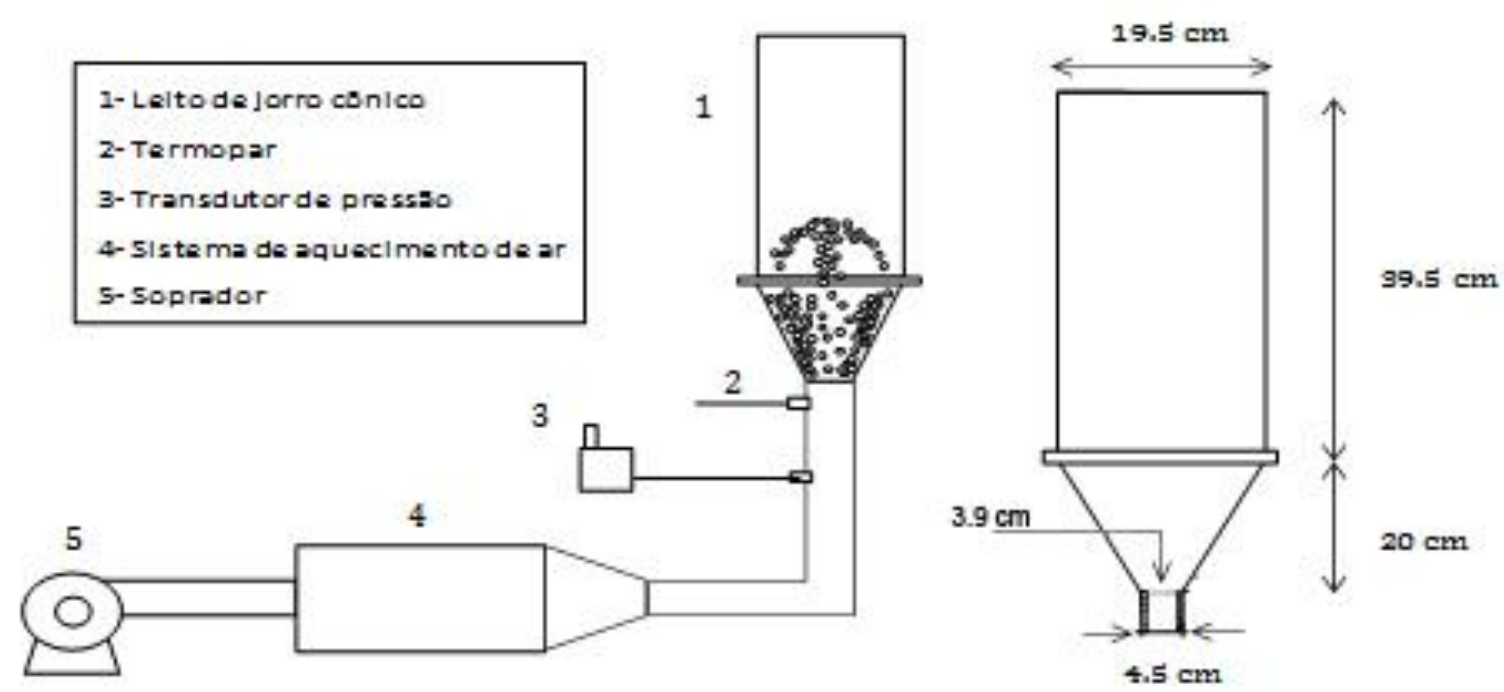

Figura 2 - Esquema do aparato experimental em escala piloto do PPGEN/UFES

\subsection{Procedimento Experimental}

Primeiramente, o leito de jorro foi preenchido com a mistura de areia e compósito AL/PEBD até atingir a altura de leito estático igual a $18 \mathrm{~cm}$. $\mathrm{O}$ ar foi, então, injetado e a vazão de ar foi regulada para um valor, aproximadamente, $10 \%$ superior ao correspondente ao regime de mínimo jorro. A temperatura do ar foi mantida constante na entrada, através do sistema de aquecimento, em torno de 27 ${ }^{\circ} \mathrm{C}$.

A vazão do ar foi reduzida em $0,5 \mathrm{~m} 3 / \mathrm{min}$. Após esperar por 5 minutos, para o processo atingir a estabilidade, a queda de pressão, determinada pelo transdutor, e a altura de fonte, medida através de papel milimetrado faceando a coluna, foram registradas. Este procedimento se repete até que a vazão de ar seja insuficiente para manter o regime de jorro do material particulado. Assim, a velocidade anterior registrada foi tomada como a velocidade de mínimo jorro. Continuou-se a reduzir a vazão de ar, contudo, em intervalos de $5 \mathrm{~m} 3 /$ min e apenas a queda de pressão foi registrada. 
Para permitir melhor reprodutibilidade dos experimentos e melhor confiabilidade estatística, este procedimento foi realizado em triplicata para cada composição de mistura.

\section{RESULTADOS E DISCUSSÕES}

$\mathrm{Na}$ figura 3, observa-se o adimensional de queda de pressão em função do adimensional de velocidade do ar (baseada no diâmetro de entrada da coluna) obtidos pelo método de velocidade do ar decrescente. Analisando os dados, na figura 3, nota-se que, para todas as composições de mistura estudadas, ocorre um aumento da queda de pressão no momento em que a velocidade é reduzida abaixo da sua velocidade de mínimo jorro $(\mathrm{v} / \mathrm{vmj}<1)$. Isto ocorre porque a velocidade de ar na entrada não é mais suficiente para manter o regime de jorro, fazendo com que as partículas retomem a condição estática, ao menos na superfície. Essa mudança abrupta de regime bloqueia a passagem de ar, o que implica no aumento da queda de pressão. Tal comportamento é característico do processo de transição entre o regime de mínimo jorro e leito estático.

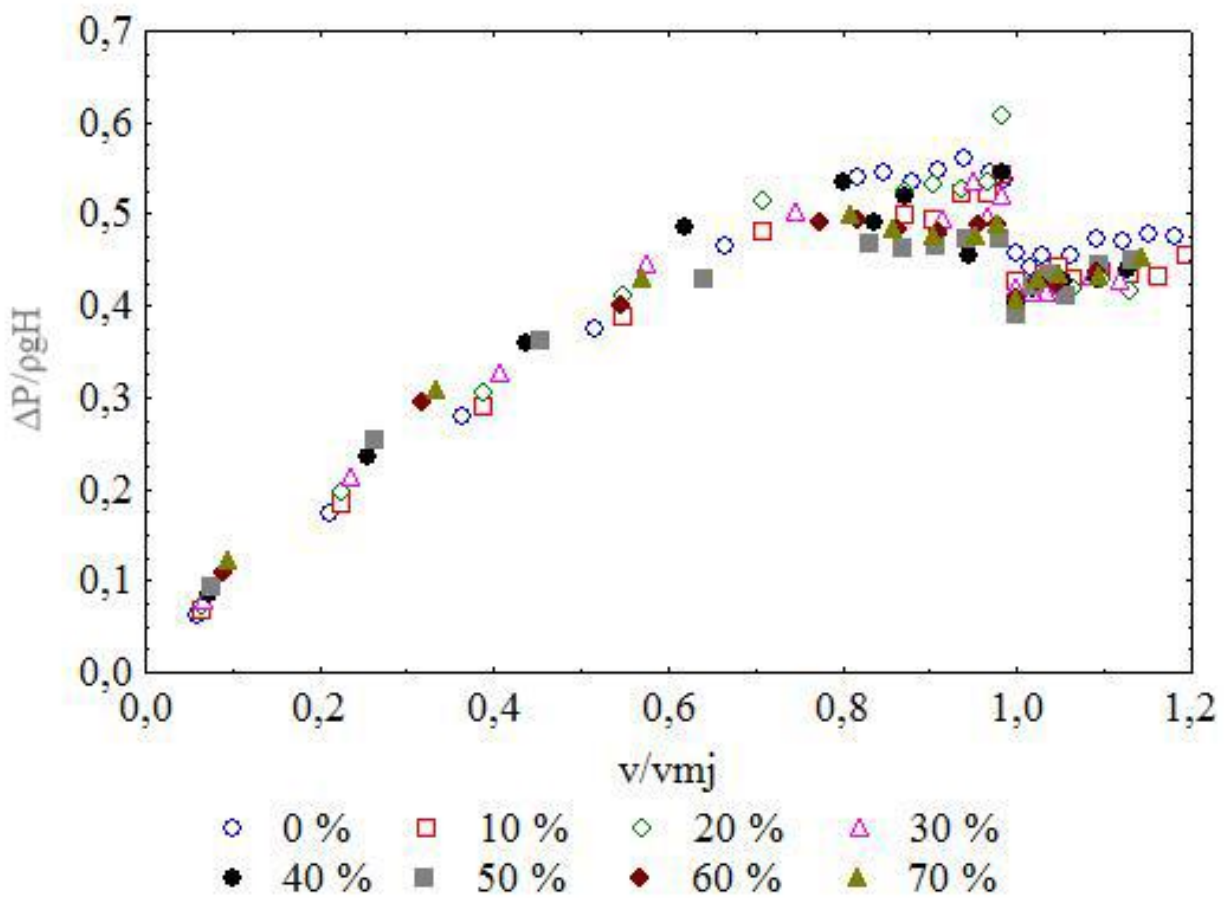

Figura 3 - Adimensional de queda de pressão em função do adimensional de velocidade do ar na entrada obtido pelo método decrescente parametrizado de acordo com a fração mássica da mistura

Experimentalmente, para todas as composições de mistura estudadas, na condições de mínimo jorro, a análise dos valores absolutos de queda de pressão no leito e velocidade do ar (não evidenciados aqui) revelaram que a velocidade e a queda de pressão no leito diminuem significativamente com o aumento da concentração de partículas de compósito AL/PEBD na mistura. Isso indica que, quando mais compósito na mistura, menor será a velocidade necessária e menor será a potência de operação requerida para operação em um de pirólise contínuo. 
De acordo com Bacelos e Freire (2006), com respeito a estabilidade do regime de mínimo jorro, esta pode ser evidenciada pela análise da flutuação da queda de pressão no leito no domínio do tempo. Tal metodologia usa a análise comportamento do desvio padrão da queda de pressão no leito em função da velocidade adimensional. Desta maneira, nota-se na figura 4 que, para os leitos analisados, o desvio padrão da queda de pressão em função da velocidade do ar não varia significativamente no regime de jorro ( $\mathrm{v} \geq \mathrm{vmj}$ ). Tal desvio apresentado está dentro da precisão de medida do transdutor de pressão. Entretanto, para misturas com concentração de compósito de Al/PEBD de $60 \%$ e $70 \%$ (dado não mostrados na Figura 4), para a condição de transição de regime de jorro e leito fixo não é observado um elevação abrupta do desvio de queda de pressão, caracterizando claramente a mudança de regime de escoamento. Visualmente, é observado que a fonte de partículas não é mantida no centro da coluna, causando assim a instabilidade na condição de mínimo jorro. Este feito pode resultar numa pirólise não uniforme e, consequentemente, reduzir a qualidade do produto.

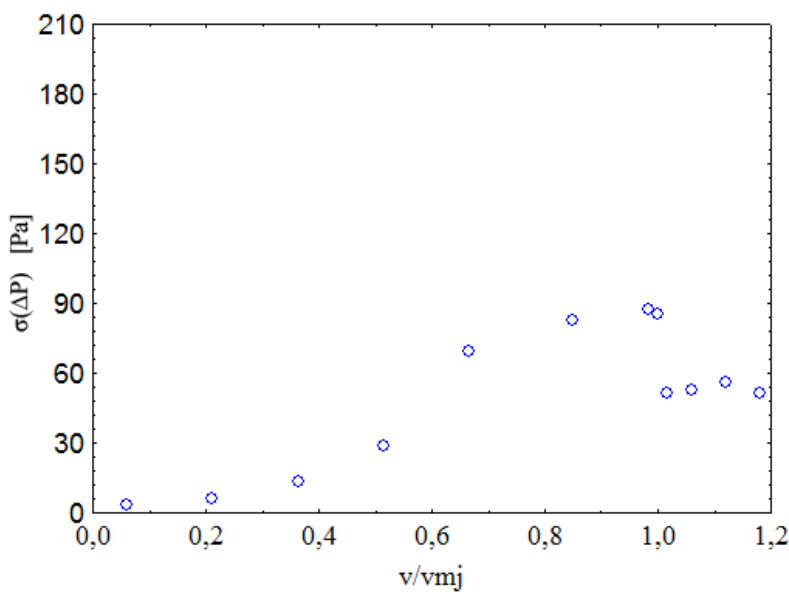

(a)

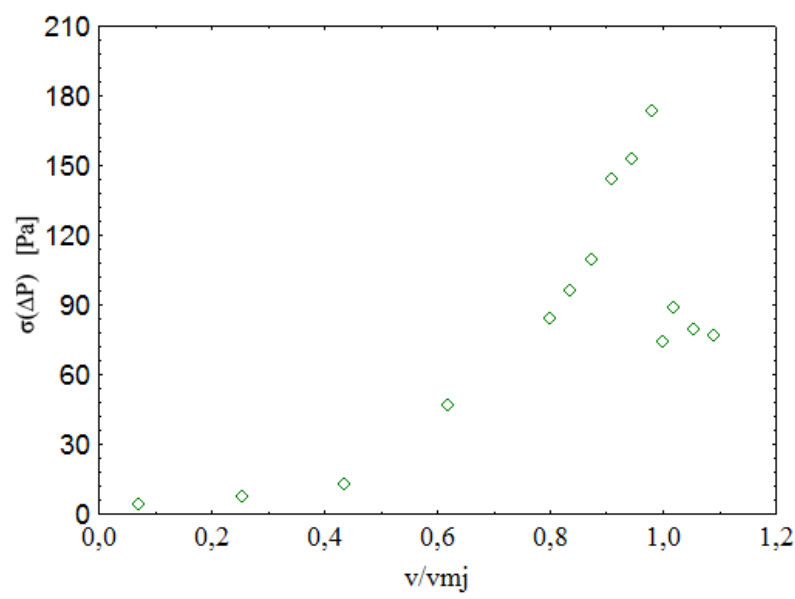

(c)

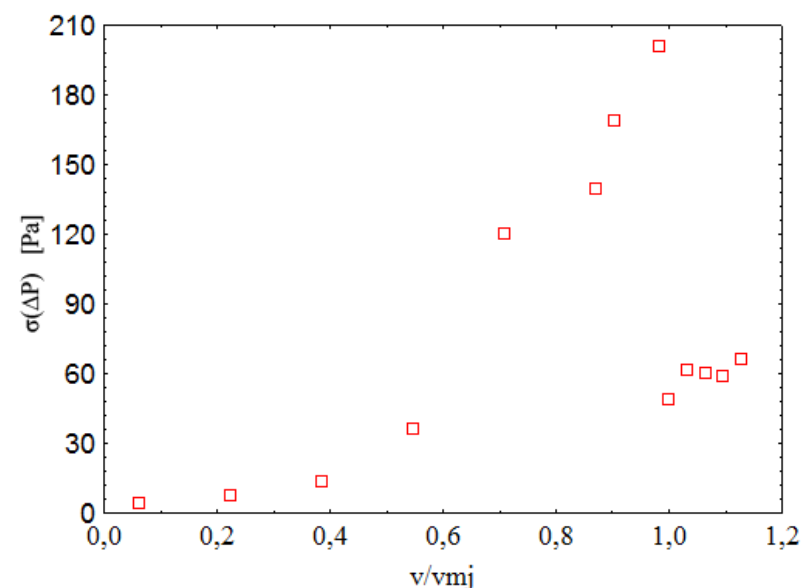

(b)

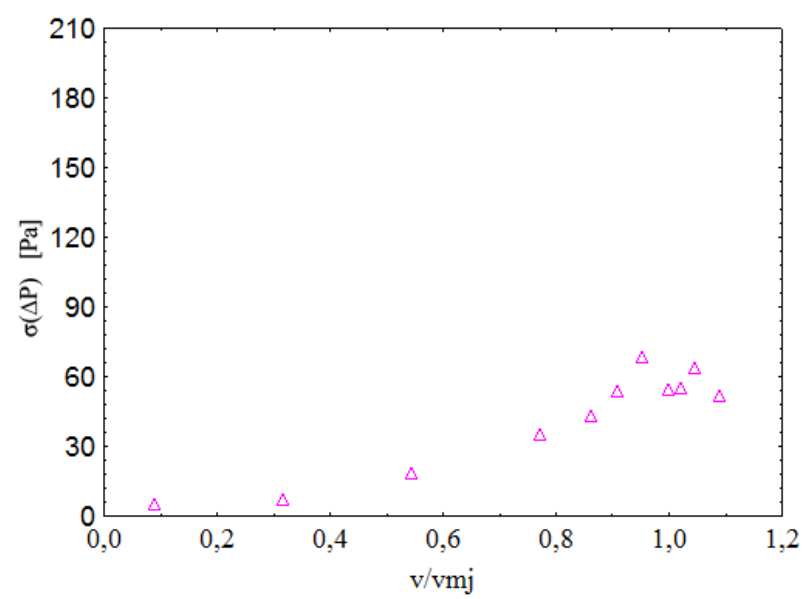

(d)

Figura 4 - Desvio padrão da queda de pressão em função do adimensional de velocidade do ar parametrizado para frações mássicas de compósito AL/PEBD igual a (a) 0, (b) 20, (c) 40 e (d) $60 \%$ 


\section{CONCLUSÃO}

Com base na análise dos dados apresentados nesta pesquisa pode-se concluir que: estável;

- o leito de jorro composto por partículas de compósito de AL/PEBD apresenta regime de jorro

- para os leitos com misturas com concentração até 50\% de areia e compósito de Al/PEBD o regime de mínimo jorro estável é evidenciado, e

- a velocidade e queda de pressão diminuem com o aumento da concentração de compósito de AL/PEBD.

\section{AGRADECIMENTOS}

Os autores agradecem ao CNPQ e a CAPES pelo apoio financeiro na realização desta pesquisa. e, também, a Tetra Pak pela concessão das amostras de compósito AL/PEBD.

\section{REFERÊNCIAS}

ABREU, M. Reciclagem de embalagens cartonadas Tetra Pak para alimentos líquidos. Tetra Pak Internacional, p. 38-43, 2002.

BACELOS, M. S. Análise do comportamento da fluidodinâmica do leito de jorro com misturas de tamanho de partículas. Tese (doutorado em engenharia química). Universidade Federal de São Carlos, São Paulo, 2006.

BACELOS, M. S.; FREIRE, J. T. Stability of spouting regimes in conical spouted beds with inert particle mixtures. Indrustrial \& Engineering Chemistry Research, v. 42 (2), p. 808-817, 2006.

BI, H. T.; MACCHI, A.; CHAOUKI, J.; LEGROS, R. Minimum spouting velocity of conical spouted beds. Canadian Journal of Chemical Engineering, v. 75, p. 460-465, 1997.

BUEKENS, A. G.; HUANG, H. Catalytic plastics cracking for recovery of gasoline-range hydrocarbons from municipal plastic wastes. Resources Conservation and Recycling, v. 23, p.163-181, 1998.

CHOI, M.; MEISEN, A. Hydrodynamics of shallow, conical spouted beds. Canadian Journal of Chemical Engineering, v. 61, p. 916-924, 1992.

HAYDARY, J.; SUSA, D.; DUDÁŠ, J. Pyrolysis of aseptic packages (Tetra Pak) in a laboratory screw type reactor and secondary thermal/catalytic tar decomposition. Waste Management, v. 33 (5), p. 11361141, 2013.

KORKMAZ. A.; YANIK, J.; BREBU, M.; VASILE, C. Pyrolysis of the Tetra Pak. Waste Management, v. 29, p. 2836-2841, 2009. 
MARQUES, I. I. D. R. Investigação do leito de jorro como reator em potencial de pirólise de partículas cartonadas. Dissertação (mestrado em energia). Universidade Federal do Espírito Santo, São Mateus, 2013.

MARQUES, I. I. D. R.; ROCHA, S. M. S.; BACELOS, M. S. Análise da fluidodinâmica de resíduos cartonados em leito de jorro. XIX Congresso Brasileiro de Engenharia Química. Búzios, 2012.

MELO, J. L. Z.; MARQUES, I. I. D. R.; BACELOS, M. S. Fluidodinâmica de compósito de polietileno e alumínio em leito de jorro. XXXVI Congresso Brasileiro de Sistemas Particulados. Maceió, 2013 (no prelo).

OLAZAR, M.; SAN JOSE, M. J.; AGUAYO, A. T.; ARANDES, J. M.; BILBAO, J. Stable operation conditions for gas-solid contact regimes in conical spouted beds. Industrial \& Engineering Chemistry Research, v. 31, p. 1784-1792, 1992.

WU, S. W. M.; LIM, C. J.; EPSTEIN, N. Hydrodynamics of spouted beds at elevated temperatures. Chemical Engineering Commum, v. 62, p. 261-268, 1987.

ZUBENS, F. V.; NEVES, F. L. Recycling of aluminum and polyethylene presente in Tetra Pak packages. São Paulo, 1999. 